Article

\title{
Polyetheretherketone Hybrid Composites with Bioactive Nanohydroxyapatite and Multiwalled Carbon Nanotube Fillers
}

\author{
Chen Liu ${ }^{1}$, Kai Wang Chan ${ }^{1}$, Jie Shen ${ }^{2}$, Cheng Zhu Liao ${ }^{3}$, Kelvin Wai Kwok Yeung ${ }^{2}$ \\ and Sie Chin Tjong ${ }^{1, *}$ \\ 1 Department of Physics and Materials Science, City University of Hong Kong, Tat Chee Avenue, Kowloon, \\ Hong Kong, China; cliu266-c@my.cityu.edu.hk (C.L.); kaiwchan8-c@my.cityu.edu.hk (K.W.C.) \\ 2 Department of Orthopedics and Traumatology, Li Ka Shing Faculty of Medicine, The University of Hong Kong, \\ Hong Kong, China; jayjayson909@gmail.com (J.S.); wkkyeung@hku.hk (K.W.K.Y.) \\ 3 Department of Materials Science and Engineering, South University of Science and Technology of China, \\ Shenzhen 518055, China; liaocz@sustc.edu.cn \\ * Correspondence: aptjong@cityu.edu.hk; Tel.: +86-852-3442-7702
}

Academic Editor: Patrick van Rijn

Received: 25 October 2016; Accepted: 2 December 2016; Published: 8 December 2016

\begin{abstract}
Polyetheretherketone (PEEK) hybrid composites reinforced with inorganic nanohydroxyapatite (nHA) and multiwalled carbon nanotube (MWNT) were prepared by melt-compounding and injection molding processes. The additions of nHA and MWNT to PEEK were aimed to increase its elastic modulus, tensile strength, and biocompatibility, rendering the hybrids suitable for load-bearing implant applications. The structural behavior, mechanical property, wettability, osteoblastic cell adhesion, proliferation, differentiation, and mineralization of the PEEK/nHA-MWNT hybrids were studied. X-ray diffraction and SEM observation showed that both nHA and MWNT fillers are incorporated into the polymer matrix of PEEK-based hybrids. Tensile tests indicated that the elastic modulus of PEEK can be increased from 3.87 to 7.13 GPa by adding 15 vol \% nHA and $1.88 \mathrm{vol} \%$ MWNT fillers. The tensile strength and elongation at break of the PEEK/(15\% nHA)-(1.88\% MWNT) hybrid were $64.48 \mathrm{MPa}$ and $1.74 \%$, respectively. Thus the tensile properties of this hybrid were superior to those of human cortical bones. Water contact angle measurements revealed that the PEEK/(15\% nHA)-(1.88\% MWNT) hybrid is hydrophilic due to the presence of nHA. Accordingly, hydrophilic PEEK/(15\% nHA)-(1.88\% MWNT) hybrid promoted the adhesion, proliferation, differentiation, and mineralization of murine MC3T3-E1 osteoblasts on its surface effectively on the basis of cell culture, fluorescence microscopy, MTT assay, WST-1 assay, alkaline phosphatase activity, and Alizarin red staining tests. Thus the PEEK/(15\% nHA)-(1.88\% MWNT) hybrid has the potential to be used for fabricating load-bearing bone implants.
\end{abstract}

Keywords: polyetheretherketone; nanohydroxyapatite; multiwalled carbon nanotube; hybrid nanocomposites; hydrophilic surface; osteoblasts; biocompatibility

\section{Introduction}

Nowadays, there is a substantial increase in the demand of bone implants due to an increase in the numbers of patients suffering from traffic injury, aging, and bone disease [1]. The technological development of biomaterials with excellent biocompatibility for bone replacements is considered of critical importance. Metallic implants such as titanium alloy, CoCrMo alloy, and 316L stainless steel have been widely used for fabricating load-bearing hip implants and bone fixation devices due to their superior mechanical strength and ductility. However, metallic implants suffer from corrosion due to the release of metal ions by interacting with body fluid containing $0.9 \% \mathrm{NaCl}$. Some ions (e.g., $\mathrm{Ni}^{2+}$, 
$\mathrm{Cr}^{3+}$ and $\left.\mathrm{Co}^{2+}\right)$ can induce allergy, inflammation and cytotoxicity [2-4]. In addition, chloride anions can attack passive films formed on metallic surfaces, leading to crevice and pitting corrosion $[5,6]$. Metallic implants also experience a stress shielding effect due to a mismatch in stiffness between the implants and host bone, resulting in a loss of bone density. In this respect, ceramics and polymers have been investigated as possible bone substitute materials. In particular, hydroxyapatite $\left[\mathrm{Ca}_{10}\left(\mathrm{PO}_{4}\right)_{6}(\mathrm{OH})_{2}\right]$ is of particular interest due to its resemblance to the mineral component of human bones. However, hydroxyapatite (HA) cannot be used for load-bearing implants due to its brittle nature. Thus inorganic HA in the form of particulates with sizes of several micrometers (mHA) are employed as reinforcing fillers for high-density polyethylene (HDPE) [7]. The inclusion of bioactive mHA fillers (40 vol \%) renders HDPE with excellent bioactivity and biocompatibility.

Polymers with good processability and light weight exhibit beneficial effects for biomedical applications [8,9]. Degradable polymers with low mechanical strength such as polylactic acid, polyglycolic acid, and their blends are typically used for bone tissue engineering applications $[10,11]$. By contrast, non-degradable polymer such as polyetheretherketone (PEEK) with high temperature durability, excellent radiation stability and high Young's modulus has found clinical applications as trauma fixation devices and spinal cages $[12,13]$. However, PEEK is bio-inert and hydrophobic, and cannot osteo-integrate with adjacent bone tissues upon implantation [14]. The bioactivity of PEEK can be greatly enhanced either by depositing HA coating on its surface [15], or by incorporating inorganic HA whiskers [16] and mHA particulates into its matrix [17]. In the latter case, Abu Bakar et al. added large mHA particulates up to $40 \mathrm{vol} \%$ into PEEK through melt compounding and injection-molding. They found that the tensile modulus of PEEK/mHA composites rises with increasing mHA content. However, the tensile strength of the composites decreases markedly as the mHA content increases. This implies that the tensile strength of PEEK/mHA composites is lower than that of pure PEEK. This is due to poor interfacial bonding between the fillers and PEEK. Further, large mHA particulates often serve as stress concentrators, thereby fracturing into small fragments during tensile testing.

Recent progress in nanotechnology research has led to the development and synthesis of novel nanomaterials with unique properties. Nanomaterials generally possess higher mechanical strength and biocompatibility compared to their micron-sized counterparts. Nanohydroxyapatite (nHA) can be synthesized by solid state reaction, co-precipitation, hydrothermal method, sol-gel process, etc. [18]. Synthetic nHA enhances protein adsorption, thereby promoting osteoblast adhesion, proliferation, and differentiation [19]. Bone tissue is a biocomposite consisting of a collagen matrix and nHA. Human cortical bone possesses an elastic modulus in the range of 7-30 GPa, tensile strength of 50-150 MPa and fracture elongation of 1\%-3\% [20]. Therefore, polymer/nHA composites have been investigated for making load-bearing hip prostheses and bone tissue scaffolds [21-26]. The additions of nHA to polypropylene (PP) of low tensile stiffness and strength improve its tensile performance moderately. The elastic modulus of PP/nHA nanocomposites is still much lower than that of cortical bone [21]. In this respect, PEEK with higher tensile stiffness and strength than PP is a potential matrix material of biocomposites for forming load-bearing bone implants.

Ma et al. prepared PEEK/nHA nanocomposites through vibrating ball-mill mixing followed by injection molding [27]. They found that that the elastic modulus of PEEK reaches a maximum value of $4.6 \mathrm{GPa}$ by adding $40 \mathrm{wt} \%(21.5 \mathrm{vol} \%) \mathrm{nHA}$. However, the modulus of this nanocomposite is much smaller than the lower limit for the modulus of cortical bone, i.e., 7 GPa. Very recently, we incorporated 4.4-21.5 vol \% nHA rods to PEEK for enhancing its mechanical stiffness and biocompatibility [23]. The results showed that the tensile modulus of PEEK-based composites increases with increasing nHA content. It increases from 3.86 GPa (pure PEEK) to a maximum value of $7.85 \mathrm{GPa}$ by adding $21.5 \mathrm{vol} \%$ (40 wt \%) nHA. The elastic modulus of PEEK/( $21.5 \mathrm{vol} \% \mathrm{nHA})$ nanocomposite reaches $7.85 \mathrm{GPa}$, being slightly higher than the lower bound in elastic modulus of cortical bone. However, PEEK/(21.5 vol \% nHA) nanocomposite is very brittle having a fracture elongation of only $0.63 \%$. These results reveal that the PEEK/( $21.5 \mathrm{vol} \% \mathrm{nHA})$ nanocomposite is unsuitable for making load-bearing implants due to its brittleness. To address this issue, carbon 
nanofibers (CNFs) of 1.6-1.9 vol \% are added to the PEEK/nHA composites for enhancing their stiffness. So PEEK/(15 vol \% nHA)-(1.9 vol \% CNF) biocomposite achieves a maximum elastic modulus of $6.54 \mathrm{GPa}$, being smaller than that of cortical bone.

As recognized, carbon nanotubes possess extraordinary high tensile modulus and strength. Multiwalled carbon nanotubes (MWNTs) possess an elastic modulus of $0.9 \mathrm{TPa}$ and tensile strength of $150 \mathrm{GPa}$ [28]. Thus MWNTs are effective nanofillers for biocomposites as they promote the adhesion and proliferation of osteoblasts on their surfaces [29,30]. By incorporating into polymers, MWNTs enhance the mechanical property and biocompatibility of resulting composites. Thus hybridizing inorganic nHA with MWNTs can effectively generate PEEK-based nanocomposites with good tensile stiffness and strength as well as excellent biocompatibility for biomedical load-bearing applications.

\section{Experimental Section}

\subsection{Materials}

PEEK-Optima (molecular weight 20,800 g/mol) pellets and MWNTs were bought from Invibio Company and Nanostructured \& Amorphous Materials Inc. (Los Alamos, NM, USA), respectively. Nanohydroxyapatite (nHA) powders were purchased from Nanjing Emperor Nano Materials (Nanjing, China). Transmission electron microscopy (TEM; Philips CM20, Philips, Amsterdam, The Netherlands) micrograph revealed nHA exhibiting a width of $20 \mathrm{~nm}$ and a length of $100 \mathrm{~nm}$ (Figure 1). PEEK pellets and nHA were dried in an oven at $55^{\circ} \mathrm{C}$ overnight prior to melt-compounding.

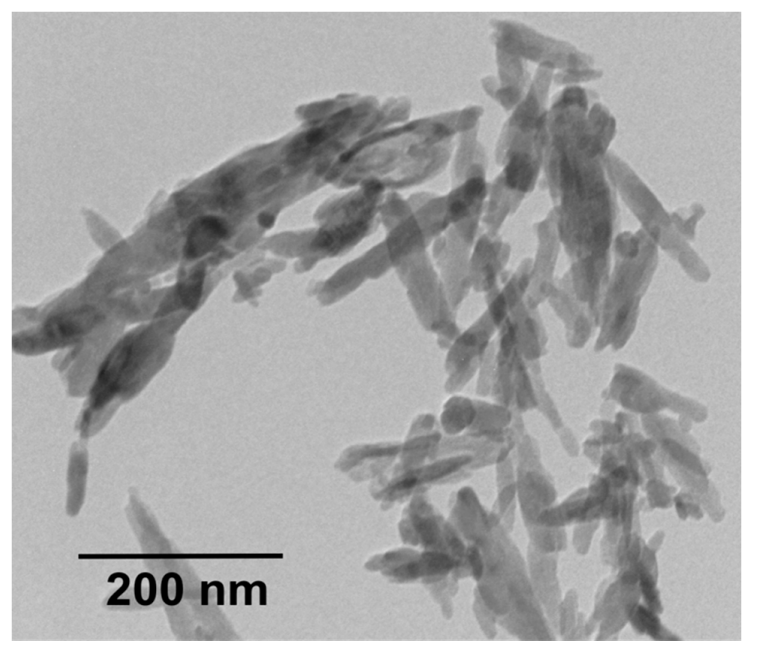

Figure 1. Transmission electron microscopy (TEM) image of nanohydroxyapatite (nHA).

\subsection{Preparation of Nanocomposites}

PEEK/(15 vol \% nHA)-MWNT hybrids with chemical compositions as listed in Table 1 were fabricated by melt-mixing and injection molding. For comparison, pure PEEK and binary PEEK/MWNT nanocomposites were also prepared. The compositions of binary PEEK/MWNT and ternary PEEK/(15 vol \% nHA)-MWNT nanocomposite in this article were expressed in volume percentage. From our previous work [23], elastic modulus and fracture strain of the PEEK/(15 vol \% nHA) nanocomposite were $6.2 \mathrm{GPa}$ and $2.71 \%$, respectively. Thus the tensile performance of this composite can be further improved by adding MWNTs. Accordingly, the nHA content of hybrid nanocomposites in this study was fixed at $15 \mathrm{vol} \%$.

In the melt-mixing process, dried PEEK pellets and nHA were initially mixed in an extruder (C.W. Brabender Instruments, South Hackensack, NJ, USA) at a screw rotation speed of $30 \mathrm{rpm}$ for $45 \mathrm{~min}$. The mixing temperatures of Brabender from hopper to extrusion die were maintained at 360-380-390-395-380-360 ${ }^{\circ} \mathrm{C}$, respectively. The extrudates were granulized by a pelletizer and 
loaded into Brabender again for second mixing under the same conditions. The purpose was to achieve homogeneous dispersion of nanofillers in the polymer matrix. The extruded products were pelletized again, dried overnight in an oven, and finally fed into an injection molder (Toyo TI-50H, Toyo Machinery \& Metal Co., Akashi, Japan) to make dog-bone tensile bars and circular disks. The disks were mainly used for cell culture, cell viability, and alkaline phosphatase activity measurements.

Table 1. The compositions of binary PEEK/MWNT and ternary PEEK/(15 vol \% nHA)-MWNT nanocomposites.

\begin{tabular}{ccccc}
\hline \multirow{2}{*}{ Sample } & \multicolumn{2}{c}{ MWNT } & \multicolumn{2}{c}{ nHA } \\
\cline { 2 - 5 } & vol \% & wt $\%$ & vol \% & wt \% \\
\hline PEEK & 0 & 0 & 0 & 0 \\
PEEK/(0.93\% MWNT) & 0.93 & 1.5 & 0 & 0 \\
PEEK/(1.88\% MWNT) & 1.88 & 3.0 & 0 & 0 \\
PEEK/(15\% nHA)-(0.93\% MWNT) & 0.93 & 1.5 & 15 & 30 \\
PEEK/(15\% nHA)-(1.88\% MWNT) & 1.88 & 3.0 & 15 & 30 \\
\hline
\end{tabular}

\subsection{Material Characterization}

X-ray diffraction (XRD) patterns of the composite specimens were obtained using a Bruker D2 Phaser X-ray diffractometer (Karlsruhe, Germany) equipped with $\mathrm{Cu}-\mathrm{K} \alpha$ radiation $(\lambda=0.154 \mathrm{~nm})$ at $30 \mathrm{kV}$. The patterns were recorded from $10^{\circ}$ to $60^{\circ}$. The morphologies of injection molded composite specimens were examined in a field-emission SEM (Jeol JSM 7100F; Tokyo, Japan). The composites were dipped in liquid nitrogen and then fractured by a hammer. The fractured surfaces were then coated with a thin carbon film.

Differential scanning calorimetry (DSC) tests were determined with a TA Instruments model 2910 (TA Instruments, New Castle, DE, USA) under a protective nitrogen atmosphere. The previous thermal history of the specimens was removed by an initial heating cycle of $20{ }^{\circ} \mathrm{C} / \mathrm{min}$ from ambient to $350^{\circ} \mathrm{C}$, followed by holding for $3 \mathrm{~min}$ at this temperature. The specimens were then cooled at a rate of $20^{\circ} \mathrm{C} / \mathrm{min}$ and the cooling traces were recorded accordingly.

\subsection{Tensile Tests}

Tensile tests were performed at room temperature using an Instron tester (model 5567, Instron Corp., Norwood, MA, USA) at a crosshead speed of $10 \mathrm{~mm} / \mathrm{min}$. The elastic modulus of PEEK and its nanocomposites was determined from the linear region of stress-strain curves. Five samples of each composition were tested, and the average values were reported.

\subsection{Water Contact Angle Tests}

Water contact angles of nanocomposite specimens were measured using Rame Hart 500-F1 advanced goniometer equipped with DROP image software (Rame-Hart Instrument Co., Succasunna, NJ, USA). Each test run was performed by producing a water droplet and its subsequent spreading into a spherical cap on the specimen surface.

\subsection{Cell Culture}

Murine MC3T3-E1 pre-osteoblasts were cultured in Dulbecco's Modified Eagle's Medium (DMEM, Thermo Scientific, Pittsburgh, PA, USA) supplemented with 10\% fetal bovine serum, penicillin, and streptomycin. Injection molded disks were sliced into small round shapes for cell cultivation and proliferation measurements. These samples were ground with $\mathrm{SiC}$ papers of different grades, followed by rinsing with $70 \%$ ethanol and phosphate buffer saline (PBS) solutions. Rinsed samples were placed in a 96-well plate. Afterwards, a cell suspension was pipetted at $10^{4}$ cells per well. The 96 -well plate was incubated in a humidified atmosphere of $5 \% \mathrm{CO}_{2} / 95 \%$ air at $37^{\circ} \mathrm{C}$ for desired periods. The culture 
medium was changed every two days. Following the incubation, the samples were washed with phosphate-buffered saline (PBS) to remove unattached cells, fixed with 10\% formaldehyde, dehydrated in a graded series of ethanol. Dehydrated cells were critical point dried and sputter deposited with gold in preparation for SEM examination. Moreover, micrographs of osteoblasts cultured on the samples were taken at day 1 and day 3 using a fluorescence microscope. Cells were washed with PBS and fixed with methanol-free formaldehyde for $30 \mathrm{~min}$ at each time point. After that, the samples were washed with PBS and stained with Alexa Fluor 488 (A12379, ThermoFisher Scientific, Carlsbad, CA, USA) at room temperature for $40 \mathrm{~min}$ to label F-actin filaments, and stained the nuclei with Hoechst 33342 (Sigma Aldrich, St. Louis, MO, USA) for $5 \mathrm{~min}$. The samples were then washed three times and stored in PBS before imaging.

\subsection{Cell Metabolic Activity}

The cell metabolic activity was determined with 3-(4,5-dimethylthiazol-2-yl)-2,5-diphenyltetrazolium bromide (MTT), and 2-(4-iodophenyl)-3-(4-nitrophenyl)-5-(2,4-disulfophenyl)-2H-tetrazolium (WST-1) assays. Rinsed samples (number of samples, $n=5$ ) were placed in a 96-well plate. A suspension of $10^{4}$ cells per well was seeded into the 96-well plate. The plate was incubated in a humidified atmosphere of $5 \%$ carbon dioxide in air at $37^{\circ} \mathrm{C}$ for 3,7 and 10 days, respectively. The culture medium was refreshed every 3 days. At days 3, 7 or 10, the medium was aspirated, then $10 \mu \mathrm{L}$ MTT solution ( $5 \mathrm{mg}$ MTT:1 mL DMEM) was added to each well and incubated for $4 \mathrm{~h}$ at $37^{\circ} \mathrm{C}$. Thus MTT reacted with mitochondrial enzyme of viable osteoblasts, producing insoluble formazan crystals. The formazan was dissolved in $10 \%$ sodium dodecyl sulfate (SDS) $/ 0.01 \mathrm{M}$ hydrochloric acid. The absorbance of dissolved formazan was measured at a wavelength of $570 \mathrm{~nm}$ using a multimode detector (Beckman Coulter DTX 880, Beckman Coulter Inc., Fullerton, CA, USA), with a reference wavelength of $640 \mathrm{~nm}$. Those wells with MTT and DMEM were used as a negative control.

For WST-1 assay, the samples $(n \geq 5)$ were first seeded with osteoblasts at $37^{\circ} \mathrm{C}$ for 3,7 and 10 days, respectively. After cell culturing for every prescribed time period, tetrazolium salt was added to each well followed by incubation for $4 \mathrm{~h}$ at $37^{\circ} \mathrm{C}$ to produce water soluble formazan. The amount of water-soluble formazan was quantified by the absorbance at $450 \mathrm{~nm}$. The cell metabolic activity of both assays was calculated by comparing the absorbance of osteoblastic cells cultured on the nanocomposite sample to that of the cells seeded on pure PEEK. The results were expressed in terms of mean \pm standard deviation (SD).

\subsection{Cellular Differentiation}

The alkaline phosphatase (ALP) activity of osteoblasts on PEEK and its nanocomposites (number of samples, $n=5$ ) was measured with a colorimetric assay kit (No. 2900-500, Stanbio Laboratory, Boerne, TX, USA). The kit used colorless 4-nitrophenyl phosphate as a substrate. The enzyme ALP of the cells hydrolyzed the substrate to color 4-nitrophenol and an inorganic phosphate. In the process, test samples were placed in each well of a 48-well plate. Osteoblasts were seeded on the samples for 3, 7 and 14 days. The culture medium was changed every 3 days. At each time period, the cells were rinsed with PBS, and lysed with $0.1 \%$ triton X-100 at $4{ }^{\circ} \mathrm{C}$ for $30 \mathrm{~min}$. The cell lysates were then centrifuged at $4{ }^{\circ} \mathrm{C}$ followed by pipetting $10 \mu \mathrm{L}$ supernatant liquid of each sample in a 96-well plate. Finally, $p$-nitrophenyl phosphate was introduced into the plate. The absorbance of $p$-nitrophenol formed was measured using a spectrophotometer (Beckman Coulter DTX 880) at $405 \mathrm{~nm}$. The ALP activity was normalized to the total protein level of the samples determined by the Bio-Rad protein Assay (Bio-Rad, Hercules, CA, USA).

Calcium mineralization of differentiating osteoblast on PEEK and its nanocomposites was measured with Alizarin red-S (Sigma Aldrich, Darmstadt, Germany) after cultivation with MC3T3-E1 pre-osteoblasts for 28 days. Disk samples of $10 \mathrm{~mm}$ diameter (number of samples, $n=5$ ) were placed in a 24-well plate. At the end of the experiment, cell cultures were fixed in $4 \%$ paraformaldehyde, washed twice with PBS and then stained with Alizarin red-S. To quantify calcium mineralization, 
the alizarin red stained cultures were incubated with 10\% cetyl-pyridinium chloride for $30 \mathrm{~min}$ in each well to release calcium-bound alizarin red into solution. Then extracted supernatant was transferred to a 96-well plate. The optical absorbance of the released alizarin red was measured at $562 \mathrm{~nm}$ using a spectrophotometer.

\subsection{Statistical Analysis for Cellular Tests}

All cell viability and differentiation assays were performed in triplicate for each treatment and the results were expressed as mean \pm standard deviation (SD). A two-way ANOVA was used to determine the significance level of difference and $p<0.05$ was considered to be statistically significant.

\section{Results and Discussion}

\subsection{Structure and Morphology}

Figure 2a shows the XRD patterns of PEEK/(15\% nHA)-(0.93\% MWNT) and PEEK/(15\% nHA)-(1.88\% MWNT) hybrids. For the purposes of comparison, XRD patterns of pure PEEK, PEEK/ $(0.93 \%$ MWNT) and PEEK/(1.83\% MWNT) composites are depicted in Figure $2 \mathrm{~b}$. Pure PEEK exhibits characteristic peaks at around $2 \theta=18.8^{\circ}, 20.7^{\circ}, 22.9^{\circ}$ and $28.9^{\circ}$, corresponding to (110), (111), (200) and (211) crystalline planes, respectively [31]. The patterns of PEEK/MWNT nanocomposites show additional peak at $26.6^{\circ}$ due to the (002) reflection of nanotubes. For both PEEK/(15\% nHA)-(0.93\% MWNT) and PEEK/ $(15 \%$ nHA)-(1.88\% MWNT) hybrids, the peaks arising from the crystalline planes of nHA fillers can be clearly observed in addition to the PEEK reflections. Moreover, the (002) peak of nHA located at $26.4^{\circ}$ overlaps with the (002) peak of MWNTs.
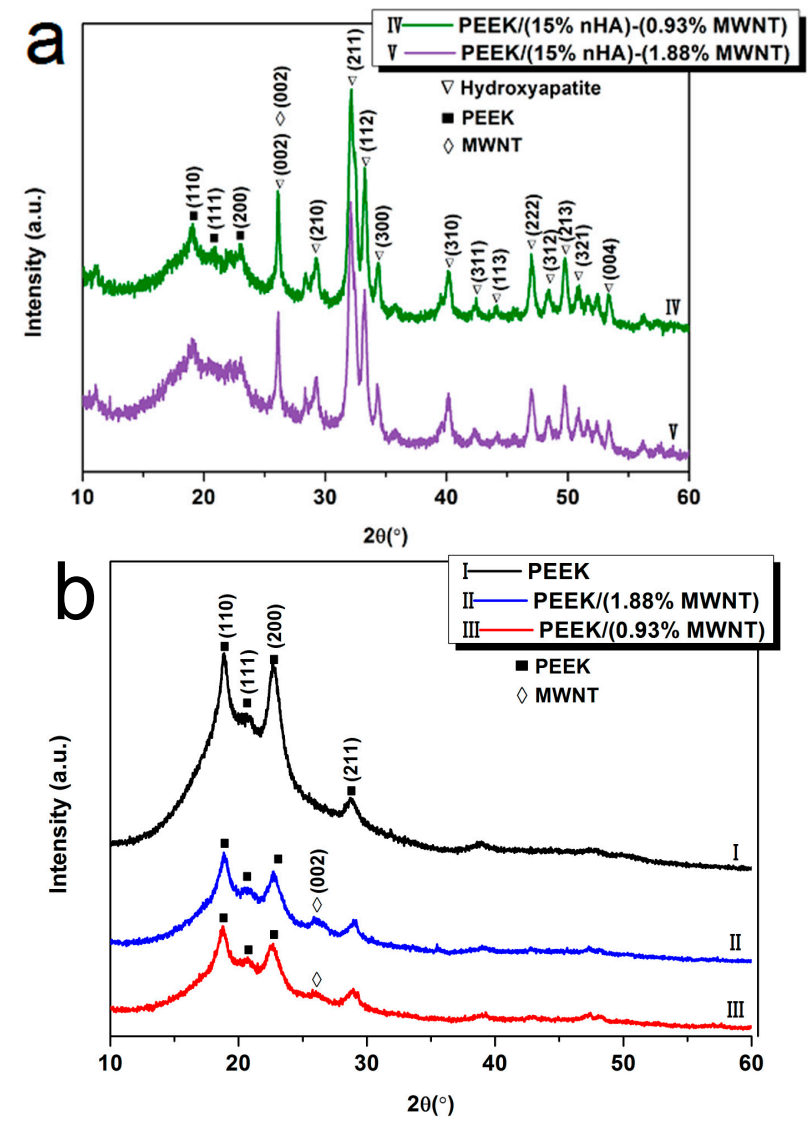

Figure 2. X-ray diffraction (XRD) patterns of (a) PEEK/(15\% nHA)-(0.93\% MWNT) and PEEK/(15\% nHA)$(1.88 \%$ MWNT) hybrids and $(\mathbf{b})$ pure PEEK and PEEK/MWNT nanocomposites. 
Figure 3a,b are the SEM images of PEEK/MWNT nanocomposites showing the homogeneous dispersion of MWNT fillers in the PEEK matrix. It can be seen that the PEEK matrix is quite rough, indicating the ductile nature of the nanocomposites. Figure 3c shows the SEM image of PEEK/(15\% nHA)-(1.88\% MWNT) hybrid. Some nHA agglomerates can be readily seen due to higher nHA filler loading in the hybrid.

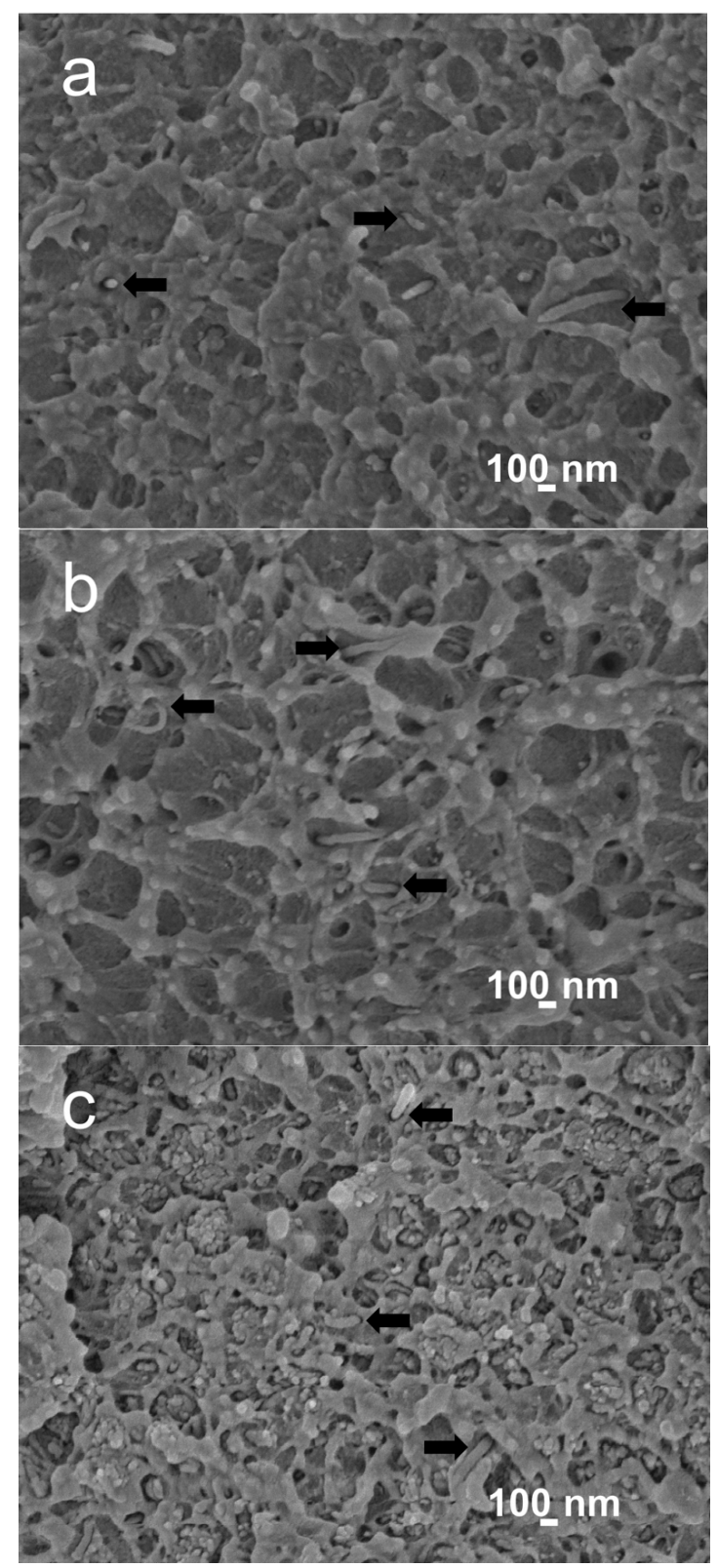

Figure 3. Scanning electron microscopy (SEM) images of (a) PEEK/(0.93 vol \% MWNT); (b) PEEK/ (1.88 vol \% MWNT) and (c) PEEK/(15\% nHA)-(1.88\% MWNT) nanocomposites. Black arrows indicate MWNT fillers.

\subsection{Crystallization Behavior}

Figure 4 shows typical DSC cooling scans of neat PEEK and its nanocomposites. The crystallization temperature $\left(T_{\mathrm{c}}\right)$ and the crystallization enthalpy $\left(\Delta H_{\mathrm{c}}\right)$ can be determined from these curves, and tabulated in Table 2 . The degree of crystallinity $\left(X_{\mathcal{c}}\right)$ of PEEK and its nanocomposites is calculated from the following equation: 


$$
X_{\mathrm{c}}(\%)=\frac{\Delta H_{\mathrm{c}}}{(1-\Phi) \times \Delta H_{\mathrm{m}}} \times 100 \%
$$

where $\Delta H_{\mathrm{m}}$ is the melting enthalpy of the $100 \%$ crystalline PEEK, i.e., $130 \mathrm{~J} / \mathrm{g}$ [31], and $\Phi$ is the weight fraction of the filler of the nanocomposites. The $X_{c}$ values of the specimens studied are also listed in Table 2. DSC results indicate that the additions of low MWNT levels to PEEK increase its crystallization temperature. The $T_{\mathrm{c}}$ value of pure PEEK is $281.14{ }^{\circ} \mathrm{C}$, and increases to 285.91 and $286.58{ }^{\circ} \mathrm{C}$ by adding $0.93 \%$ and $1.88 \%$ MWNTs, respectively. These demonstrate that MWNTs serve as effective nucleation sites for PEEK crystallites on cooling from melting temperature, leading to an increase of the degree of crystallinity. By adding 15\% nHA to the PEEK/(0.93\% MWNT) and PEEK/(1.83\% MWNT), the $T_{\mathrm{c}}$ values appear to decrease. Moreover, $X_{\mathrm{c}}$ values of PEEK $/(15 \% \mathrm{nHA})-(0.93 \% \mathrm{MWNT})$ and PEEK/(15\% nHA)-(0.93\% MWNT) hybrids also decrease due to the agglomeration of nHA fillers as shown in Figure 4.

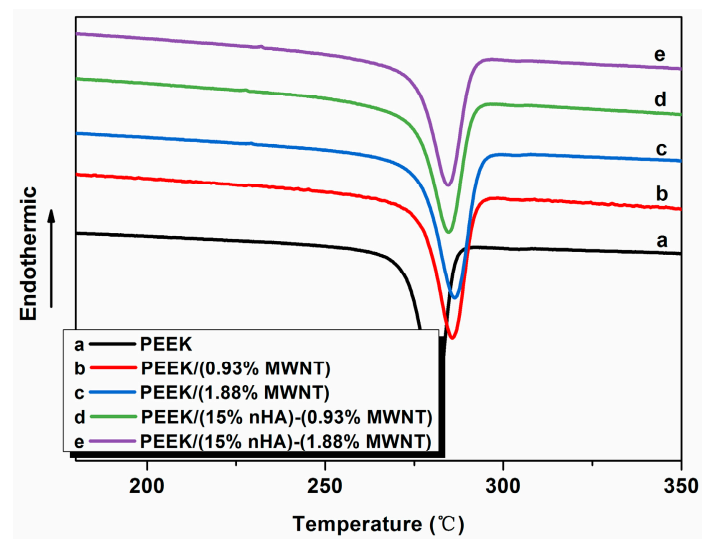

Figure 4. Differential scanning calorimetry (DSC) cooling curves of PEEK and its nanocomposites.

Table 2. Crystallization parameters of PEEK and its nanocomposites.

\begin{tabular}{ccccc}
\hline Sample & $\boldsymbol{T}_{\mathbf{c}}\left({ }^{\circ} \mathbf{C}\right)$ & $\boldsymbol{\Delta} \boldsymbol{H}_{\mathbf{c}}(\mathbf{J} / \mathbf{g})$ & $\boldsymbol{X}_{\mathbf{c}}(\mathbf{\%})$ & FWHM $\left({ }^{\circ} \mathbf{C}\right)$ \\
\hline PEEK & 281.14 & 59.38 & 45.68 & 7.57 \\
PEEK/(0.93\% MWNT) & 285.91 & 59.26 & 46.28 & 8.81 \\
PEEK/(1.88\% MWNT) & 286.58 & 59.85 & 47.46 & 9.62 \\
PEEK/(15\% nHA)-(0.93\% MWNT) & 284.90 & 36.87 & 41.40 & 9.21 \\
PEEK/(15\% nHA)-(1.88\% MWNT) & 284.73 & 35.97 & 41.30 & 9.07 \\
\hline
\end{tabular}

Furthermore, the full width at half-maximum (FWHM) of crystallization peak of PEEK increases with increasing MWNT content. The FWHM values of PEEK and its nanocomposites are also listed in Table 2. This increase in the width of the crystallization peak of PEEK/MWNT nanocomposites due to MWNT additions could be attributed to a wider crystal size distribution [32]. However, the addition of $15 \%$ nHA to the PEEK/MWNT nanocomposites leads to a slight decrease of the FWHM of crystallization peak and a marked decrease in crystallinity. Thus a large amount of nHA fillers that agglomerate in the PEEK matrix cannot act as effective sites for polymeric chain nucleation.

\subsection{Tensile Behavior}

Figure 5 shows the stress-strain curves of PEEK and its nanocomposites. The tensile parameters of these samples and human cortical bone are summarized in Table 3. It is obvious that the elastic modulus of PEEK can be increased from 3.87 to 4.25 GPa by adding 1.88 vol \% MWNTs. The elongation at break of PEEK is reduced from $67.1 \%$ to $56.48 \%$ by adding 1.88 vol $\%$ MWNTs. However, the PEEK/(15\% nHA)-(1.88\% MWNT) hybrid still exhibits ductile feature as evidenced by the SEM micrograph as shown in Figure $3 b$. MWNTs with exceptionally high stiffness also 
exhibit high mechanical flexibility upon the application of large deformation strains [33]. Thus the additions of low MWNT contents to PEEK enhance its tensile stiffness and strength, with a small reduction in tensile ductility. On the other hand, the addition of $15 \mathrm{vol} \%$ nHA to PEEK increases it stiffness to $6.2 \mathrm{GPa}$, but with a drastic reduction in fracture elongation to $2.71 \%$ due to the brittle nature of nHA [23]. From Table 3, the addition of 1.88 vol \% MWNT to PEEK/(15\% nHA) leads to a further increase in elastic modulus from 6.20 to $7.13 \mathrm{GPa}$. The tensile strength and elongation at break of the PEEK/(15\% nHA)-(1.88\% MWNT) hybrid are $64.48 \mathrm{MPa}$ and $1.74 \%$, respectively. Therefore, all the tensile properties of PEEK/(15\% nHA)-(1.88\% MWNT) hybrid are superior to those of human cortical bone. This demonstrates that the PEEK/(15\% nHA)-(1.88\% MWNT) hybrid can be used for fabricating load-bearing bone implants in terms of mechanical property consideration. From Table 2, PEEK/(15\% nHA)-(0.93\% MWNT) and PEEK/(15\% nHA)-(1.88\% MWNT) hybrids exhibit lower $X_{\mathrm{c}}$ values comparing with binary PEEK/MWNT composites. As a result, PEEK/(15\% nHA)-(0.93\% MWNT) and PEEK/(15\% nHA)-(1.88\% MWNT) hybrids exhibit lower tensile strength than binary PEEK/MWNT composites as shown in Table 3. As recognized, polymers and their composites with higher degree of crystallinity exhibit better tensile strength due to the presence of more ordered polymer crystallites.

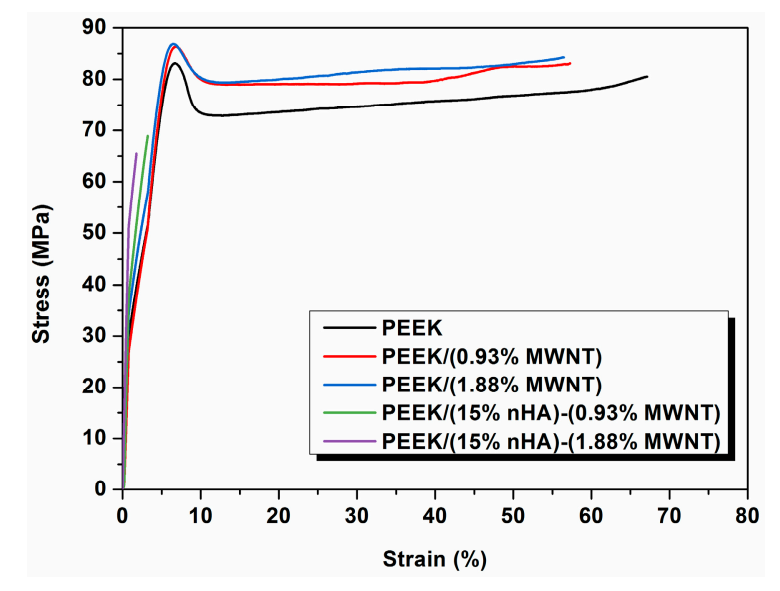

Figure 5. Tensile stress-strain curves of PEEK and its nanocomposites.

Table 3. Tensile properties of PEEK and its nanocomposites.

\begin{tabular}{cccc}
\hline Sample & Elastic modulus (GPa) & Tensile stress (Mpa) & Elongation (\%) \\
\hline PEEK & $3.87 \pm 0.10$ & $80.06 \pm 0.49$ & $67.10 \pm 13.40$ \\
PEEK/(0.93\% MWNT) & $4.21 \pm 0.11$ & $83.38 \pm 0.78$ & $57.25 \pm 13.20$ \\
PEEK/(1.88\% MWNT) & $4.25 \pm 0.85$ & $82.08 \pm 3.68$ & $56.48 \pm 24.90$ \\
PEEK/(15\% nHA)-(0.93\% MWNT) & $6.83 \pm 0.20$ & $70.99 \pm 7.51$ & $2.32 \pm 1.15$ \\
PEEK/(15\% nHA)-(1.88\% MWNT) & $7.13 \pm 0.12$ & $64.48 \pm 8.51$ & $1.74 \pm 0.58$ \\
PEEK/(15\% nHA) [23] & $6.20 \pm 0.13$ & $70.56 \pm 3.22$ & $2.71 \pm 0.34$ \\
Human cortical bone [20] & $7-30$ & $50-150$ & $1-3$ \\
\hline
\end{tabular}

\subsection{Wettability}

Contact angle is a measure of the wettability of a solid when a liquid comes in contact with it. A hydrophobic solid surface forms when the water contact angle is larger than $90^{\circ}$. In this case, the water droplet does not spread but rather forms a spherical cap resting on the solid surface with a contact angle. By contrast, the hydrophilic solid surface shows a water contact angle smaller than $90^{\circ}$. Figure 6 shows the appearances of water droplets and contact angles on the surfaces of all samples studied. Pure PEEK displays a contact angle of $106.58^{\circ} \pm 1.13^{\circ}$, revealing its hydrophobic nature. Adding $0.93 \mathrm{vol} \%$ and $1.88 \mathrm{vol} \%$ MWNTs to PEEK decreases its water contact angles. At $1.88 \mathrm{vol} \%$ MWNT loading, the contact angle reduces to $99.07^{\circ} \pm 0.50^{\circ}$. However, contact angle drops markedly 
to $84.08^{\circ} \pm 0.65^{\circ}$ and $76.99^{\circ} \pm 0.78^{\circ}$ by hybridizing $15 \%$ nHA with $0.93 \%$ MWNTs and $1.88 \%$ MWNTs, respectively. This is due to the presence of nHA, which is hydrophilic in nature. Thus hydrophilic PEEK/(15\% nHA)-(0.93\% MWNT) and PEEK/(15\% nHA)-(1.88\% MWNT) hybrids favor adhesion of osteoblasts on their surfaces.

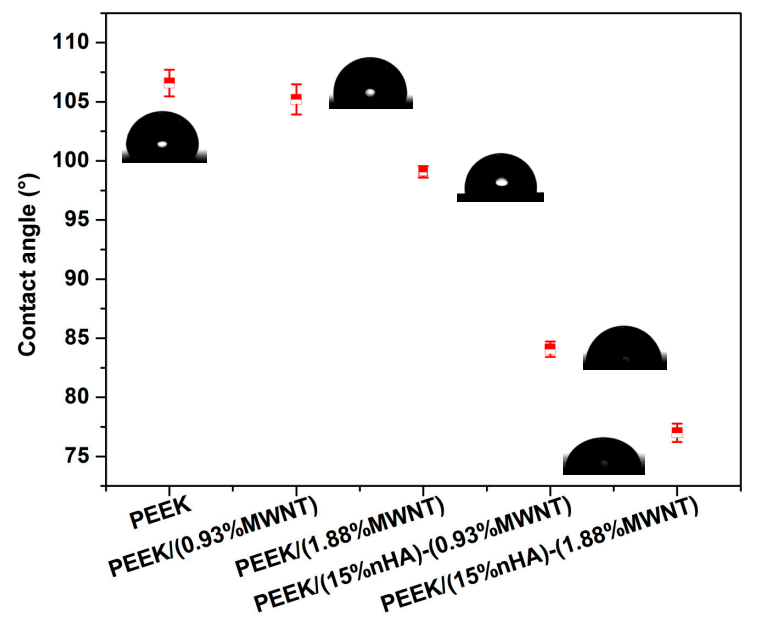

Figure 6. Contact angles formed by spreading of water droplets on PEEK and its nanocomposites.

From the literature, enhancing the hydrophilicity of the polymer surface leads to increased cell spreading and adhesion. Upon implantation into the body, water molecules interact with the material surfaces followed by protein adsorption. These surface proteins play a key role in cell adhesion, proliferation, and subsequent differentiation [34,35]. The bound proteins on the material surface provide recognition sites for cell adhesion via specific cell receptors such as integrins $[35,36]$.

\subsection{Cellular Adhesion}

The initial cell-biomaterial interactions mimic to a certain extent the natural communication of cells with extracellular matrix (ECM). Cell adhesion is mediated by the integrins of cell surface receptor and the ECM proteins (e.g., fibronectin, collagen), which in turn are sensitive to the chemical composition and wettability of biomaterials [37]. Integrins are transmembrane proteins that bind ECM and interact with the cytoskeleton at focal adhesion complexes. Once integrins adhere to such molecules, they are activated and clustered into nascent adhesions [37,38]. Therefore, osteoblast adhesion on the biomaterial surface is evidenced by markedly expedited cell spreading and dense actin fiber formation. PEEK exhibits a relatively hydrophobic surface, thereby limiting cell attachment. By adding bioactive nHA and MWNTs, the resulting PEEK hybrids become hydrophilic as discussed above. Figure 7a-c shows the fluorescence micrographs of osteoblasts cultured on the PEEK/(15\% nHA)-(0.93\% MWNT) hybrid for 1 and 3 days, respectively. These micrographs show extensive spreading of osteoblasts and distinct actin stress fibers, especially for hybrids cultivated with osteoblasts for 3 days. Furthermore, more distinct actin stress fibers are observed on the PEEK/(15\% nHA)-(1.88\% MWNT) hybrid as expected (Figure $8 \mathrm{a}, \mathrm{b})$. The higher expression level of actin and focal adhesion contacts demonstrate enhanced cellular activity and stable cytoskeleton, thereby facilitating progressive development of cellular processes [39]. For comparison, fluorescence micrographs of osteoblasts cultured on the PEEK/(0.93\% MWNT) and PEEK/(1.88\% MWNT) nanocomposites for 3 days are shown in Figure 9a,b, respectively. These images reveal that osteoblast can attach and spread on the PEEK/MWNT composite surfaces in the absence of nHA, but with lower amounts of the cell adhesion. It is noted that these fluorescence figures provide qualitative observations only. 


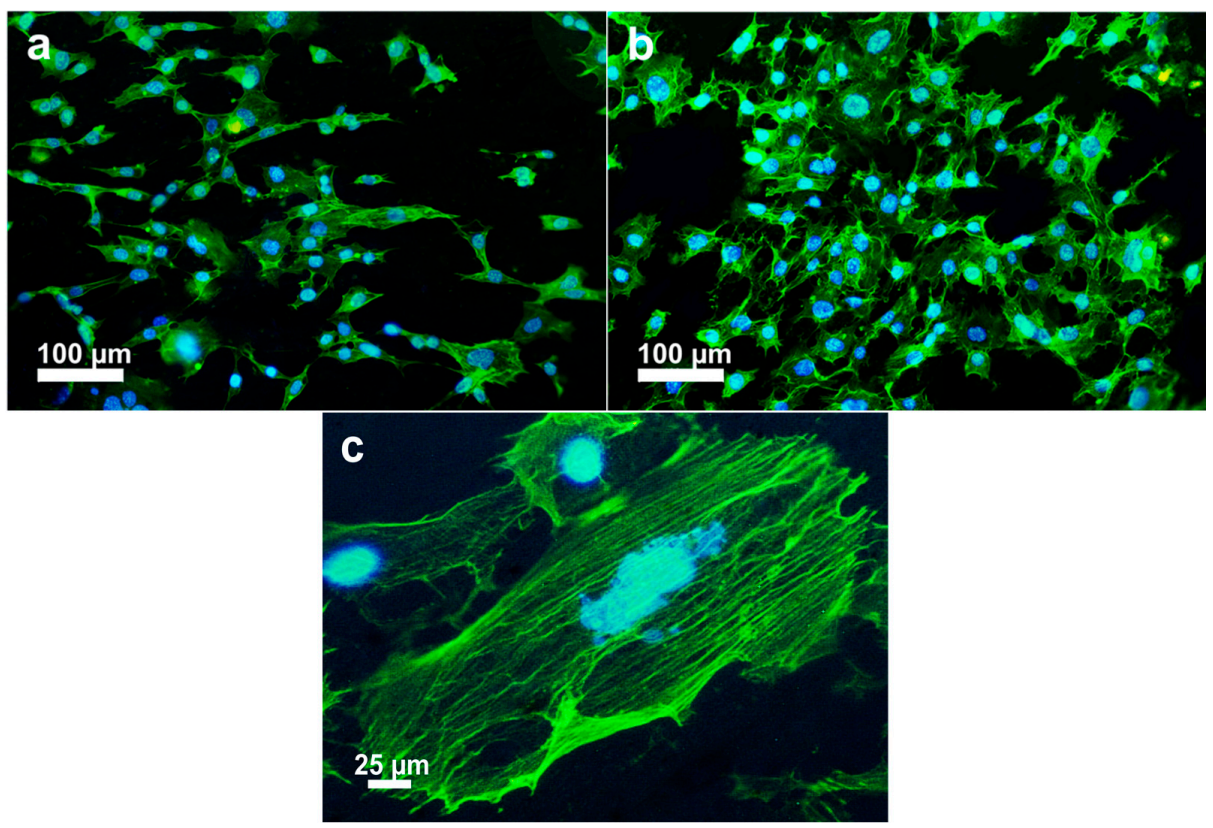

Figure 7. Fluorescence micrographs of osteoblasts cultured on PEEK/(15\% nHA)-(0.93\% MWNT) hybrid and stained for F-actin cytoskeleton (green) and nucleus (blue) for (a) 1 day and (b) 3 days; (c) High magnification image showing the morphology of well-organized actin filaments of a cell at day 3.

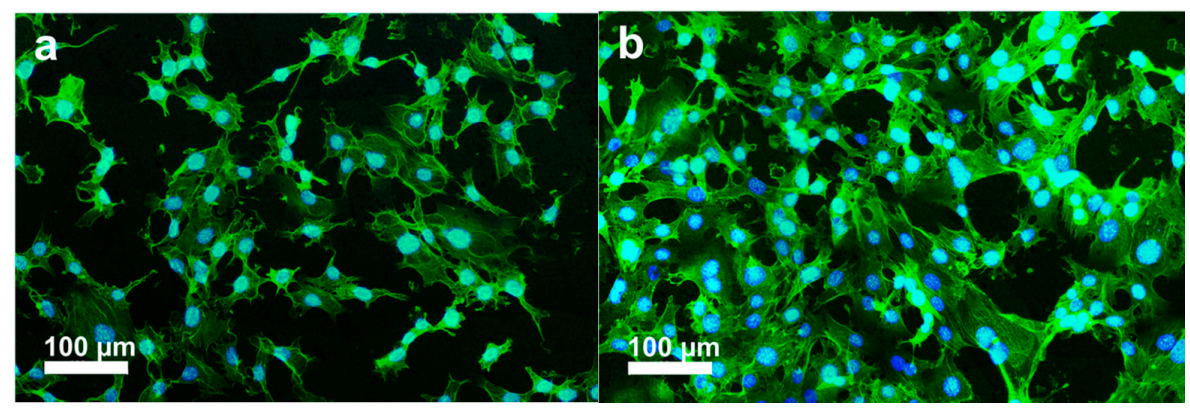

Figure 8. Fluorescence micrographs of osteoblasts cultured on PEEK/(15\% nHA)-(1.88\% MWNT) hybrid and stained for F-actin cytoskeleton (green) and nucleus (blue) at (a) day 1 and (b) day 3.
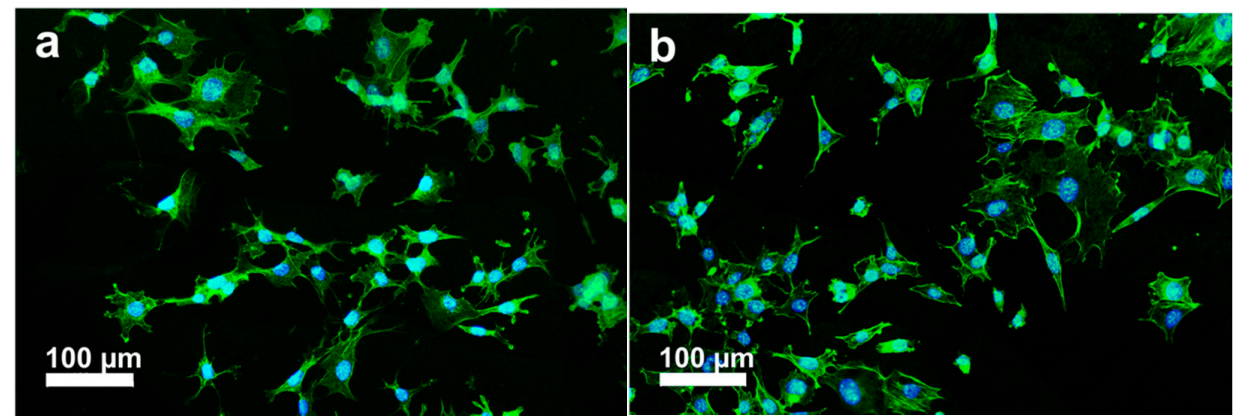

Figure 9. Fluorescence micrographs of osteoblasts cultured on (a) PEEK/(0.93\% MWNT) and (b) PEEK/(1.88\% MWNT) nanocomposites and stained for F-actin cytoskeleton (green) and nucleus (blue) for 3 days.

The adherence and spreading of osteoblasts on a material surface can also be examined through SEM imaging. Figure 10a,b show the SEM micrographs of PEEK/(1.88\% MWNT) nanocomposites seeded with osteoblasts for 2 and 4 days, respectively. Apparently, osteoblasts attach firmly on these 
nanocomposite surfaces after cultivation for 2 days. At day 4 , they then proliferate and spread flatly on the surfaces in which many neighbor cells link with each other through cytoplasmic extension. The PEEK/ $(1.83 \%$ MWNT) nanocomposite surface is almost covered with osteoblasts after culturing for 4 days. It is evident that MWNTs act as effective sites for osteoblastic adhesion. Interestingly, osteoblasts attach and spread entirely on the PEEK/ $(15 \%$ nHA)-( $0.93 \%$ MWNT $)$ hybrid surface cultured for 2 days (Figure 11a). At day 4, the osteoblastic feature remains unchanged (Figure 11b).

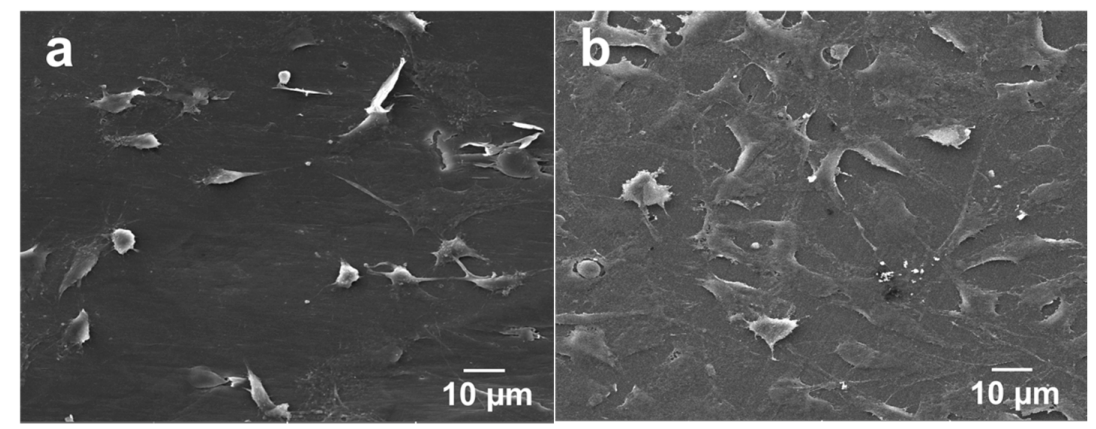

Figure 10. SEM images of PEEK/(1.88\% MWNT) nanocomposite cultured with osteoblasts for (a) 2 and (b) 4 days.

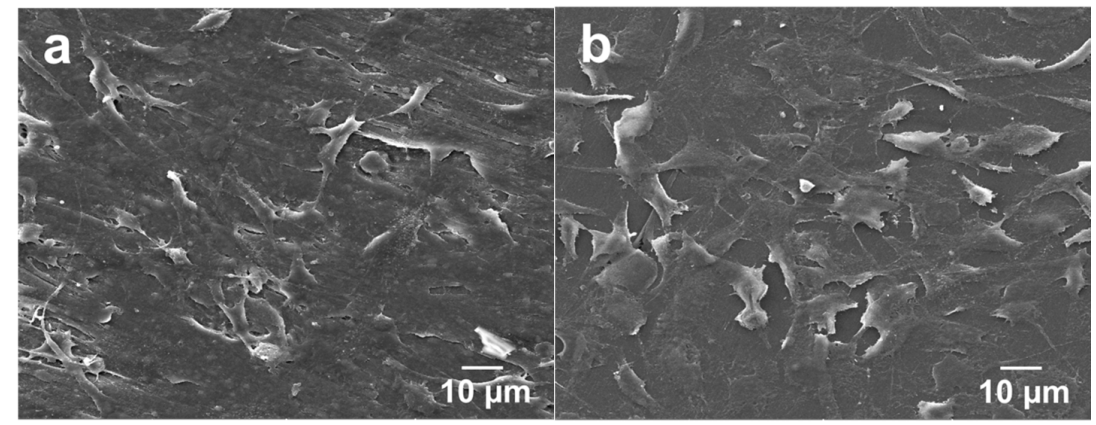

Figure 11. SEM images of PEEK/(15\% nHA)-(0.93\% MWNT) hybrid cultured with osteoblasts for (a) 2 and (b) 4 days.

\subsection{Cellular Metabolic Activity}

The MTT and WST-1 assays can measure viable cell number [40], while Mwenifumbo et al. demonstrated that MTT assay is related to cell metabolic activity [41]. A live/dead stain would be used to measure cell viability. Figure 12 shows the results of MTT measurements for PEEK and its nanocomposites. PEEK/(0.93\% MWNT) and PEEK/(1.88\% MWNT) composites exhibit higher cell metabolic activity comparing to pure PEEK specimen, especially after cultivation for 7 days. Thus MWNT fillers promote the growth of osteoblasts on the nanocomposite surfaces. It has been reported in the literature that carbon nanotubes may exhibit cytotoxic effects towards biological cells $[42,43]$. The cytotoxicity is mainly caused by individual carbon nanotubes dispersed in the cell culture medium or organic suspensions. As such, individual carbon nanotubes can penetrate cell membrane and reside in the cytoplasm [44,45]. On the other hand, no cytotoxic effects are observed in carbon nanotube fillers of polymer nanocomposites [21-23]. This is because carbon nanotube fillers are embedded firmly within the matrix of polymer nanocomposites. In this case, they serve as the active sites for the attachment of osteoblasts. From Figure 12, a marked increase in cell metabolic activity can be seen in both PEEK/(15\% nHA)-(0.93\% MWNT) and PEEK/(15\% nHA)-(1.88\% MWNT) hybrids. Thus the presence of inorganic nHA in such hybrids offers a beneficial effect in enhancing their biocompatibility. From the water contact measurements, these hybrids are hydrophilic due to the presence of nHA. On the contrary, PEEK/ $(0.93 \%$ MWNT) and PEEK/(1.83\% MWNT) composites 
exhibit hydrophobic behavior because of the absence of nHA. As discussed above, increased surface hydrophilicity enhances protein adsorption and cell spreading. Thus hydrolytic materials favor the adhesion and proliferation of osteoblasts on their surfaces [34-46].

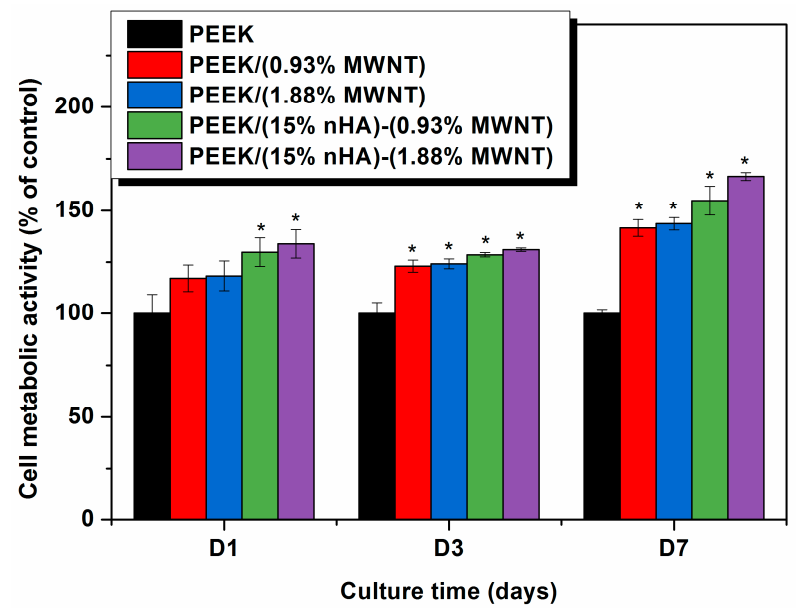

Figure 12. 3-(4,5-Dimethylthiazol-2-yl)-2,5-diphenyltetrazolium bromide (MTT) assay results showing cell metabolic activity of murine MC3T3-E1 pre-osteoblasts grown on PEEK and its nanocomposites for 1,3 and 7 days. ${ }^{*}$ represents $p<0.05$. Significance is assessed for each time-point assayed.

It is worth-noting that MTT measurements tend to give lower cellular metabolic activity for carbon nanotubes due to the formation of insoluble formazan products [47]. MTT assay is a colorimetric test based on the reduction of the yellowish, water-soluble tetrazolium salt into a water-insoluble, purple formazan product by mitochondrial enzyme succinate dehydrogenase of living cells in the culture. The reduction occurs only when reductase enzymes are active. Dead cells are incapable of converting MTT into formazan. MTT assay needs an additional solubilization step to dissolve color formazan crystals. The intensity of the color gives a relative count of viable cells. Such formazan crystals do not dissolve completely in an organic solvent, particularly in the presence of carbon nanotubes [47]. The undissolved crystals in a solvent give low colorimetric readings in the spectrophotometric measurements, causing low cell metabolic activity. By contrast, WST-1 assay produces water soluble formazan and does not form insoluble clusters like MTT. Therefore, WST-1 assay generates reliable results for assessing cell metabolic activity. Figure 13 shows the cell metabolic activity measured by the WST-1 assay for the PEEK-based nanocomposites. Apparently, WST-1 assay gives considerably higher cell metabolic activity than MTT assay. At day 7, the amount of viable cells for PEEK/(15\% nHA)-(0.93\% MWNT) and PEEK/(15\% nHA)-(1.88\% MWNT) hybrids is relatively high as compared to PEEK/ $(0.93 \%$ MWNT) and PEEK/(1.83\% MWNT) composites.

For the MTT and WST assays, the amount of formazan crystals produced is directly proportional to the number of metabolically active cells in the culture. Both assays reveal that, after 7 days, cells cultured on the PEEK/(15\% nHA)-(0.93\% MWNT) and PEEK/(15\% nHA)-(1.88\% MWNT) hybrids exhibit a significantly higher metabolic activity than on the PEEK control specimen. As aforementioned, nHA enhances protein adsorption, thereby promoting osteoblast adhesion, proliferation, and differentiation [19]. In other words, proteins that can induce osteoblast adhesion, including fibronectin and vitronectin, have been reported to have higher adsorption rates on nHA [19,48]. Moreover, osteoblastic cells also attach and survive on the MWNT materials, producing higher metabolic activity on the basis of MTT results [41]. Therefore, PEEK/(15\% nHA)-(0.93\% MWNT) and PEEK/(15\% nHA)-(1.88\% MWNT) hybrid nanocomposites exhibit enhanced cell activity compared to the control PEEK specimen. 


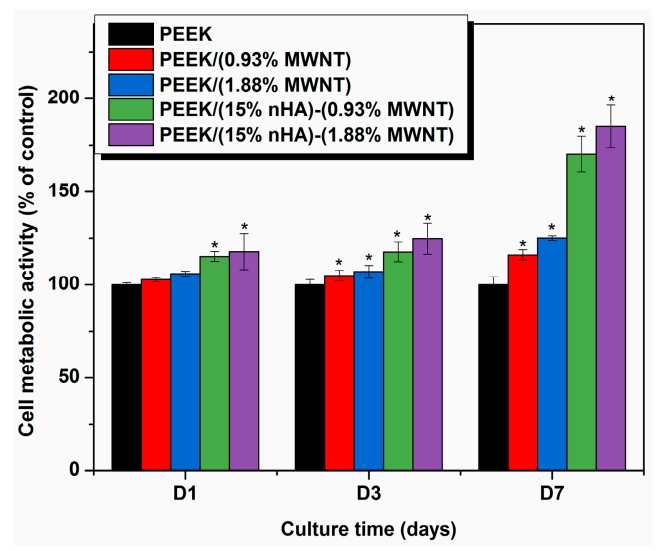

Figure 13. 2-(4-Iodophenyl)-3-(4-nitrophenyl)-5-(2,4-disulfophenyl)-2H-tetrazolium (WST-1) assay results showing cell metabolic activity of murine MC3T3-E1 pre-osteoblasts grown on PEEK and its nanocomposites for 1,3 and 7 days. ${ }^{*}$ represents $p<0.05$. Significance is assessed for each time-point assayed.

\subsection{Cellular Differentiation and Mineralization}

Alkaline phosphatase (ALP) activity and calcium mineralization are mostly used as biochemical markers for early and late differentiation of osteoblast cells, respectively $[49,50]$. Bone formation involves a series of events that occur in the initial proliferation and differentiation stages, followed by mineralization. Proliferating osteoblasts show alkaline phosphatase activity in vitro; this is greatly enhanced during early differentiation of osteoblasts. Alkaline phosphatase catalyzes hydrolysis of $p$-nitrophenyl phosphate in alkaline buffer and produces phenol and inorganic phosphate. An enhanced level of this enzyme is required just before the onset of bone mineralization, providing localized enrichment of inorganic phosphate for nHA nucleation and proliferation. Figure 14 shows the ALP activity for the osteoblastic cells exposed to PEEK and its nanocomposites. Apparently, very low ALP activity is detected after 3 days for all samples investigated. At day 14, alkaline phosphatase activity of osteoblasts grown on the hybrids, especially PEEK/(15\% nHA)-(1.88\% MWNT) increases markedly in comparison to those observed in pure PEEK and PEEK-MWNT nanocomposites. This demonstrates the ability of this hybrid nanocomposite to enhance early osteoblast differentiation.

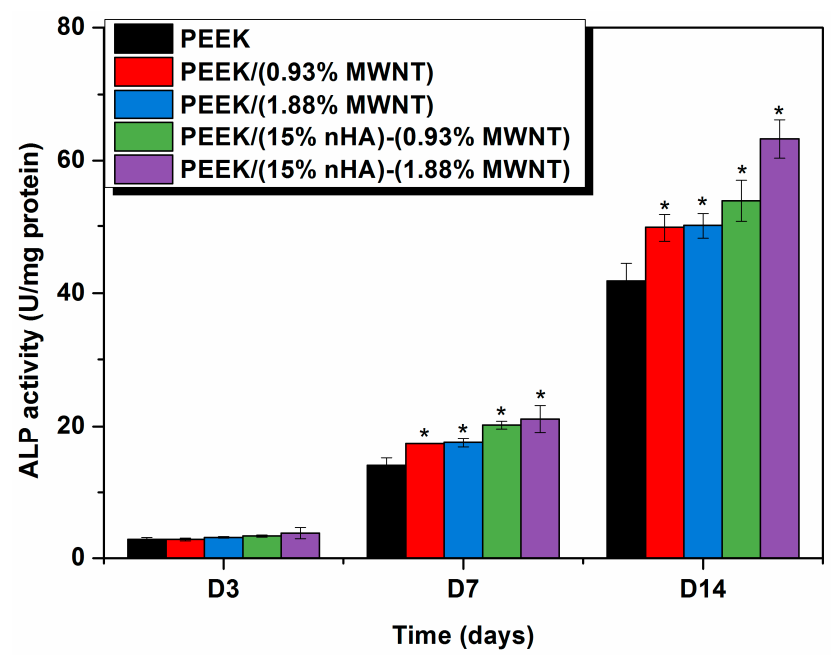

Figure 14. Alkaline phosphatase (ALP) activity normalized to protein content of murine MC3T3-E1 pre-osteoblasts cultured on PEEK and its nanocomposites for 1, 3 and 7 days. ${ }^{*}$ represents $p<0.05$.

Finally, Alizarin Red staining after 28 days of cell culture is used to determine the presence of calcium minerals on the PEEK-based nanocomposites. Bone forming cells containing calcium deposits 
are stained dark red by the Alizarin red solution. Prior to staining, white nodules can be seen on the PEEK/MWNT and PEEK/(15\% nHA)-MWNT nanocomposites. However, large calcified nodules are more abundant in the hybrids (Figure 15). White mineralized nodules were stained a dark red color by alizarin red. Mineralized nodule formation indicates the final stages of osteoblastic differentiation at 28 days. To clarify that the nHA fillers of PEEK-based hybrids do not contribute to dark red staining, PEEK/(0.93\% MWNT) composite and PEEK/(15\% nHA)-MWNT hybrids without cells are also stained with Alizarin Red (Figure 16). Apparently, these specimens do not display dark red color, indicating that the nanohydroxyapatite in the hybrids does not contribute to the staining. Figure 17 shows the optical density (OD) of calcium mineralization of MC3T3-E1 pre-osteoblasts cultured on PEEK and its nanocomposites for 28 days. Low mineral deposition level occurs in pure PEEK as expected. Increased mineral deposition is found in the PEEK/(0.93\% MWNT) and PEEK/(1.83\% MWNT) nanocomposites comparing with neat PEEK. However, high mineralization level can be observed in both hybrids, especially in the PEEK/(15\% nHA)-(1.88\% MWNT) sample. Increased mineralization in cultured osteoblasts implies increased calcium deposition. As previously mentioned, the presence of nHA in PEEK-based hybrids converts hydrophobic PEEK to hydrophilic, thereby promoting protein adhesion, and subsequent osteoblastic cell attachment, proliferation, differentiation, and eventual calcium mineralization.
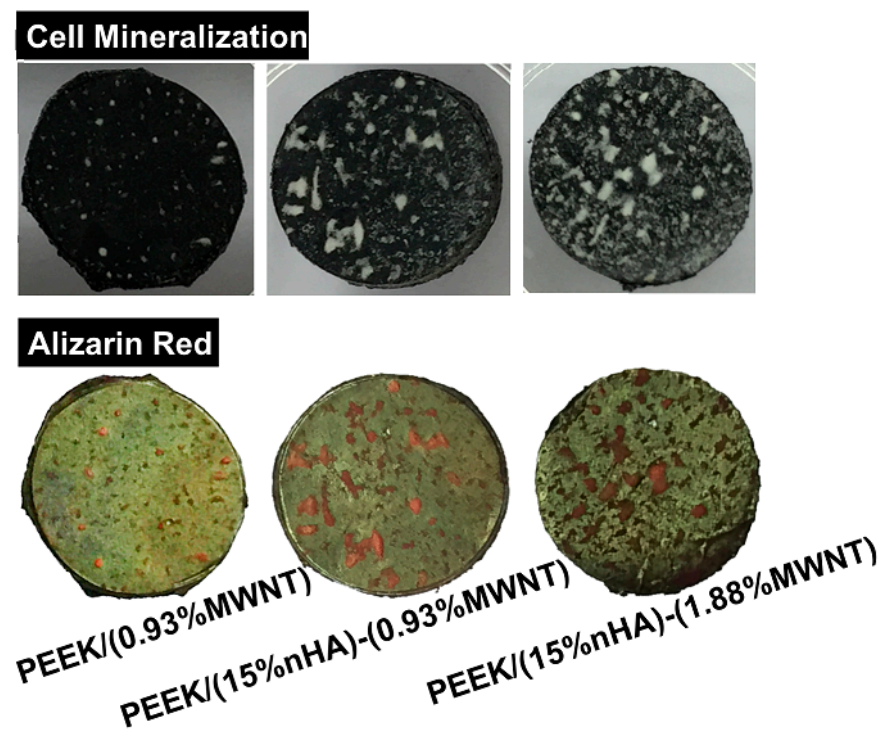

Figure 15. Representative photographs showing calcium mineralization (before staining) on PEEK/(0.93\% MWNT), PEEK/(15\% nHA)-(0.93\% MWNT) and PEEK/(15\% nHA)-(1.88\% MWNT) nanocomposites cultured with murine MC3T3-E1 pre-osteoblasts for 28 days (top); Calcified nodules appeared as a dark red color on these samples after staining with Alizarin red-S (bottom).

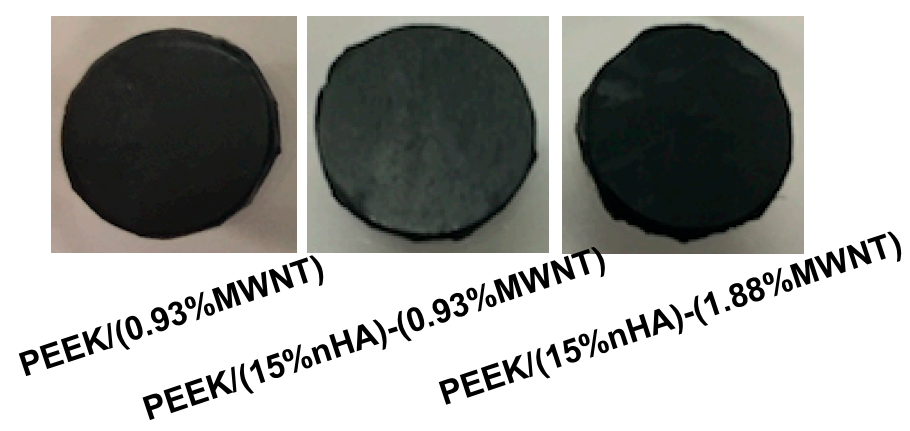

Figure 16. Photographs of Alizarin Red stained PEEK/(0.93\% MWNT), PEEK/(15\% nHA)(0.93\% MWNT) and PEEK/(15\% nHA)-(1.88\% MWNT) nanocomposites without cell exposure. 


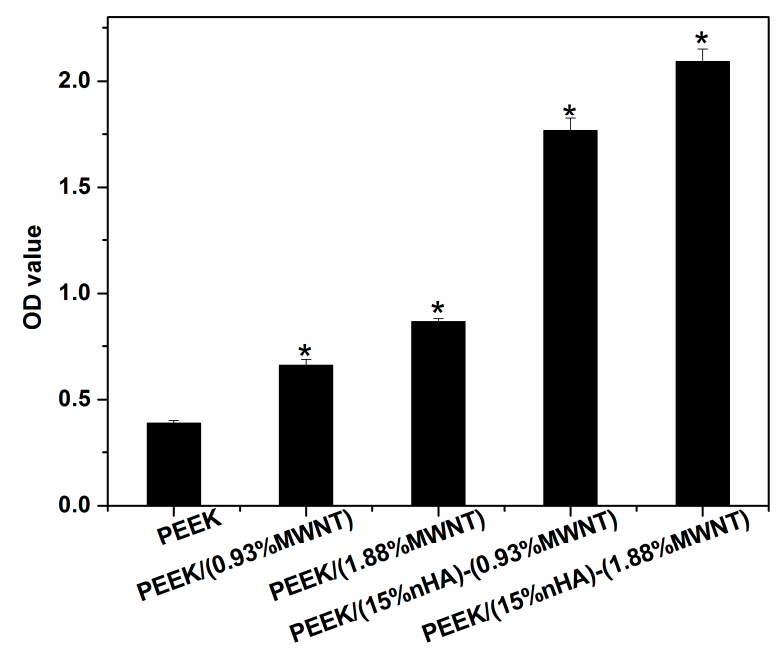

Figure 17. Optical absorbance of MC3T3-E1 pre-osteoblasts cultured on PEEK and its nanocomposites for 28 days and stained with Alizarin red. * represents $p<0.05$.

\section{Conclusions}

Melt-mixing and injection molding techniques have been successfully adapted for fabricating PEEK/(15\% nHA)-MWNT hybrid nanocomposites. Combining the advantages of inorganic nHA and MWNTs, the resulting hybrid nanocomposites show good tensile property and excellent biocompatibility. In particular, the elastic modulus of PEEK can be increased from 3.87 to $7.13 \mathrm{GPa}$ by adding $15 \mathrm{vol} \% \mathrm{nHA}$ and $1.88 \mathrm{vol} \%$ MWNT. The tensile strength and elongation at break of the PEEK/(15\% nHA)-(1.88\% MWNT) hybrid are $64.48 \mathrm{MPa}$ and $1.74 \%$, respectively. Thus the tensile properties of this hybrid are superior to those of human cortical bone. Water contact angle measurements reveal that pure PEEK is hydrophobic with a water contact angle of $106^{\circ}$. Adding $1.88 \%$ MWNTs to PEEK decreases its water contact angles to $98^{\circ}$. The contact angle drops markedly to $77^{\circ}$ by adding $1.88 \%$ MWNTs and $15 \%$ nHA hybrid fillers to PEEK. Accordingly, hydrophilic PEEK/(15\% nHA)-(1.88\% MWNT) hybrid favors the adhesion, proliferation and differentiation of osteoblasts on its surface based on the results of cell culture, fluorescence microscopy, MTT assay, WST-1 assay, ALP, and Alizarin red staining tests. Thus the PEEK/(15\% nHA)-(1.88\% MWNT) hybrid has the potential to be used for fabricating load-bearing orthopedic implants.

Acknowledgments: This work was supported by the Applied Research Grant (No. 9667126), City University of Hong Kong, Shenzhen Science and Technology Project Grant (Grant No. JCYJ20150630145302228), China and Shenzhen Knowledge Innovation Program of Basic Research Items of Guangdong Province (Grant No. JCYJ20140414090541811), China.

Author Contributions: Chen Liu carried out the drop water contact angle test, SEM examination of nanocomposite features, fluorescence microscopic imaging, MTT assay, WST-1 assay, ALP assay, and Alizarin red-S staining. Kai Wang Chan fabricated pure PEEK and composite materials using melt-compounding and injection molding processes as well as performed tensile measurements. Jie Shen performed cell culture for all assays and SEM examination of osteoblastic cells. Cheng Zhu Liao carried out XRD and DSC tests. Kelvin Wai Kwok Yeung supervised cell cultivation, MTT, WST-1, ALP, and Alizarin red-S staining tests. Sie Chin Tjong designed the project and wrote the manuscript.

Conflicts of Interest: The authors declare no conflict of interest.

\section{References}

1. Yu, X.; Tang, X.; Gohil, S.V.; Laurencin, C.T. Biomaterials for bone regenerative engineering. Adv. Healthc. Mater. 2015, 24, 1268-1285. [CrossRef] [PubMed]

2. Hallab, N.; Meritt, K.; Jacobs, J.J. Metal sensitivity in patients with orthopedic implants. J. Bone Jt. Surg. Am. 2001, 83A, 428-436. [CrossRef] 
3. Okazaki, Y.; Gatoh, E. Comparison of metal release from various metallic biomaterials in vitro. Biomaterials 2005, 26, 11-21. [CrossRef] [PubMed]

4. Scharf, B.; Clement, C.C.; Zolla, V.; Perino, G.; Yan, B.; Elci, S.G.; Purdue, E.; Goldring, S.; Macaluso, F.; Cobelli, N.; et al. Scharf Molecular analysis of chromium and cobalt-related toxicity. Sci. Rep. 2014, 4, 5729. [CrossRef] [PubMed]

5. Tjong, S.C.; Yeager, E. ESCA and SIMS studies of the passive film on iron. J. Electrochem. Soc. 1981, 128, 2251-2254. [CrossRef]

6. Tjong, S.C.; Hoffman, R.W.; Yeager, E.B. Electron and ion spectroscopic studies of the passive film on iron-chromium alloys. J. Electrochem. Soc. 1982, 129, 1662-1668. [CrossRef]

7. Huang, J.; Disilvio, L.; Wang, M.; Tanner, K.E.; Bonfield, W. In vitro mechanical and biological assessment of hydroxyapatite-reinforced polyethylene composite. J. Mater. Sci. Mater. Med. 1997, 8, 775-779. [CrossRef] [PubMed]

8. Meng, Y.Z.; Tjong, S.C. Rheology and morphology of compatibilized polyamide 6 blends containing liquid crystalline copolyesters. Polymer 1998, 39, 99-107. [CrossRef]

9. Tjong, S.C.; Liu, S.L.; Li, R.K.Y. Mechanical properties of injection moulded blends of polypropylene with thermotropic liquid crystalline polymer. J. Mater. Sci. 1996, 31, 479-484. [CrossRef]

10. Ulery, B.D.; Nair, L.S.; Laurencin, C.T. Biomedical applications of biodegradable polymers. J. Polym. Sci. B Polym. Phys. 2011, 49, 832-864. [CrossRef] [PubMed]

11. Du, L.C.; Meng, Y.Z.; Wang, S.J.; Tjong, S.C. Synthesis and degradation behavior of poly(propylene carbonate) derived from carbon dioxide and propylene oxide. J. Appl. Polym. Sci. 2004, 92, 1840-1846. [CrossRef]

12. Díez-Pascual, A.M.; Naffakh, M.; Marco, C.; Ellis, G.; Gómez-Fatou, M.A. High-performance nanocomposites based on polyetherketones. Prog. Mater. Sci. 2012, 57, 1106-1190. [CrossRef]

13. Kurtz, S.M.; Devine, J.N. PEEK biomaterials in trauma, orthopedic, and spinal implants. Biomaterials 2007, 28, 4845-4869. [CrossRef] [PubMed]

14. Ajami, S.; Coathup, M.J.; Khoury, J.; Blunn, G.W. Augmenting the bioactivity of polyetheretherketone using a novel accelerated neutral atom beam technique. J. Biomed. Mater. Res. B 2016. [CrossRef] [PubMed]

15. Lee, J.H.; Jang, H.L.; Lee, K.M.; Baek, H.R.; Jin, K.; Hong, K.S.; Noh, J.H.; Lee, H.K. In vitro and in vivo evaluation of the bioactivity of hydroxyapatite-coated polyetheretherketone biocomposites created by cold spray technology. Acta Biomater. 2013, 9, 6177-6187. [CrossRef] [PubMed]

16. Converse, G.L.; Yue, W.M.; Roeder, R.K. Processing and tensile properties of hydroxyapatite-whiskerreinforced polyetheretherketone. Biomaterials 2007, 28, 927-935. [CrossRef] [PubMed]

17. Abu Bakar, M.S.; Cheng, M.H.; Tang, S.M.; Yu, S.C.; Liao, K.; Tan, C.T.; Khor, K.A.; Cheang, P. Tensile properties, tension-tension fatigue and biological response of polyetheretherketone-hydroxyapatite composites for load-bearing orthopedic implants. Biomaterials 2003, 24, 2245-2250. [CrossRef]

18. Tjong, S.C. Advances in Biomedical Science and Engineering; Bentham: New York, NY, USA, 2009.

19. Zhang, L.; Webster, T.J. Nanotechnology and nanomaterials: Promises for improved tissue regeneration. Nano Today 2009, 4, 66-80. [CrossRef]

20. Bonfield, W.; Wang, M.; Tanner, K.E. Interfaces in analogue biomaterials. Acta. Mater. 1998, 46, $2509-2518$. [CrossRef]

21. Liao, C.Z.; Wong, H.M.; Yeung, K.W.; Tjong, S.C. The development, fabrication and material characterization of polypropylene composites reinforced with carbon nanofiber and hydroxyapatite nanorod hybrid fillers. Int. J. Nanomed. 2014, 9, 1299-1310.

22. Li, K.; Yeung, C.Y.; Yeung, K.W.; Tjong, S.C. Sintered hydroxyapatite/polyetheretherketone nanocomposites: Mechanical behavior and biocompatibility. Adv. Eng. Mater. 2012, 14, B155-B165. [CrossRef]

23. Chan, K.W.; Liao, C.Z.; Wong, H.M.; Yeung, K.W.; Tjong, S.C. Preparation of polyetheretherketone composites with nanohydroxyapatite rods and carbon nanofibers having high strength, good biocompatibility and excellent thermal stability. RSC Adv. 2016, 6, 19417-19429. [CrossRef]

24. Liu, C.; Wong, H.M.; Yeung, K.W.; Tjong, S.C. Novel electrospun polylactic acid nanocomposite fiber mats with hybrid graphene oxide and nanohydroxyapatite reinforcements having enhanced biocompatibility. Polymers 2016, 8, 287. [CrossRef]

25. Venkatesan, J.; Kim, S.K. Nano-hydroxyapatite composite biomaterials for bone tissue engineering-A review. J. Biomed. Nanotechnol. 2014, 10, 3124-3140. [CrossRef] [PubMed] 
26. Sun, F.; Zhou, H.; Lee, J. Various preparation methods of highly porous hydroxyapatite/polymer nanoscale biocomposites for bone regeneration. Acta Biomater. 2011, 7, 3813-3829. [CrossRef] [PubMed]

27. Ma, R.; Tang, S.; Tan, H.; Lin, W.; Wang, Y.; Wei, J.; Zhao, L.; Tang, T. Preparation, characterization, and in vitro osteoblast functions of a nano-hydroxyapatite/polyetheretherketone biocomposite as orthopedic implant material. Int. J. Nanomed. 2014, 9, 3949-3961.

28. Demczyk, B.G.; Wang, Y.M.; Cumings, J.; Hetman, M.; Han, W.; Zettl, A.; Ritchie, R.O. Direct mechanical measurement of the tensile strength and elastic modulus of multiwalled carbon nanotubes. Mater. Sci. Eng. A 2002, 334, 173-178. [CrossRef]

29. Smart, S.K.; Cassady, A.I.; Lu, G.Q.; Martin, D.J. The biocompatibility of carbon nanotubes. Carbon 2006, 44, 1034-1047. [CrossRef]

30. Usui, Y.; Aoki, K.; Narita, N.; Murakami, N.; Nakamura, I.; Nakamura, K.; Ishigaki, N.; Yamazaki, H.; Horiuchi, H.; Kato, H.; et al. Carbon nanotubes with high bone-tissue compatibility and bone-formation acceleration effects. Small 2008, 4, 240-246. [CrossRef] [PubMed]

31. Blundell, D.J.; Osborn, B.N. The morphology of poly(aryl-ether-ether-ketone). Polymer 1983, 24, 953-958. [CrossRef]

32. Tzavalas, S.; Mouzakis, D.E.; Drakonakis, V.; Gregoriou, V.G. Polyethylene terephthalate-multiwall nanotubes nanocomposites: Effect of nanotubes on the conformations, crystallinity and crystallization behavior of PET. J. Polym. Sci. B Polym. Phys. 2008, 46, 668-676. [CrossRef]

33. Falvo, M.R.; Clary, G.J.; Taylor, R.M.; Chi, V.; Brooks, F.P., Jr.; Washburn, S.; Superfine, R. Bending and buckling of carbon nanotubes under large strain. Nature 1997, 389, 582-584. [PubMed]

34. Lee, J.H.; Khang, G.; Lee, J.W.; Lee, H.B. Interaction of different types of cells on polymer surfaces with wettability gradient. J. Colloid Interface Sci. 1998, 205, 323-330. [CrossRef] [PubMed]

35. Arima, Y.; Iwata, H. Effect of wettability and surface functional groups on protein adsorption and cell adhesion using well-defined mixed self-assembled monolayers. Biomaterials 2007, 28, 3074-3082. [CrossRef] [PubMed]

36. Hynes, R.O. Integrins: Bidirectional, allosteric signaling machines. Cell 2002, 10, 673-687. [CrossRef]

37. Palacio, M.L.; Bushan, B. Bioadhesion: A review of concepts and applications. Philos. Trans. R. Soc. A 2012, 370, 2321-2347. [CrossRef] [PubMed]

38. Petit, V.; Thiery, J.P. Focal adhesions: Structure and dynamics. Biol. Cell 2000, 92, 477-494. [CrossRef]

39. Chen, Q.; Kinch, M.S.; Lin, T.H.; Burridge, K.; Juliano, R.L. Integrin-mediated cell adhesion activates mitogen-activated protein kinase. J. Biol. Chem. 1994, 269, 26602-26605. [PubMed]

40. Van Meerloo, J.; Kaspers, G.J.; Cloos, J. Cell sensitivity assays: The MTT assay. Methods Mol. Biol. 2011, 731, 237-245. [PubMed]

41. Mwenifumbo, S.; Shaffer, M.S.; Stevens, M.M. Exploring cellular behavior with multi-walled carbon nanotube constructs. J. Mater. Chem. 2007, 17, 1894-1902. [CrossRef]

42. Ren, H.X.; Chen, X.; Liu, J.H.; Gu, N.; Huang, X.J. Toxicity of single-walled carbon nanotube: How we were wrong? Mater. Today 2010, 13, 6-8. [CrossRef]

43. Hamilton, R.F., Jr.; Wu, Z.; Mitra, S.; Shaw, P.K.; Holian, A. Effect of MWCNT size, carboxylation, and purification on in vitro and in vivo toxicity, inflammation and lung pathology. Part. Fibre Toxicol. 2013, 10, 57. [CrossRef] [PubMed]

44. Porter, A.E.; Gass, M.; Muller, K.; Skepper, J.N.; Midgley, P.A.; Welland, M. Direct imaging of single-walled carbon nanotubes in cells. Nat. Nanotechnol. 2007, 2, 713-717. [CrossRef] [PubMed]

45. Nagai, H.; Okazaki, Y.; Chew, S.Y.; Misawa, N.; Yamashita, Y.; Akatsuka, S.; Ishihara, T.; Yamashita, K.; Yoshikawa, Y.; Yasui, H.; et al. Diameter and rigidity of multiwalled carbon nanotubes are critical factors in mesothelial injury and carcinogenesis. Proc. Natl. Acad. Sci. USA 2011, 108, E1330-E1338. [CrossRef] [PubMed]

46. Altankov, G.; Grinnell, F.; Groth, T. Studies on the biocompatibility of materials: Fibroblast reorganization of substratum-bound fibronectin on surfaces varying in wettability. J. Biomed. Mater. Res. 1996, 30, 385-391. [CrossRef]

47. Worle-Knirsch, J.M.; Pulskamp, K.; Krug, H.F. Oops they did it again! Carbon nanotubes hoax scientists in viability assays. Nano Lett. 2006, 6, 1261-1268. [CrossRef] [PubMed]

48. Tran, N.; Webster, T.J. Nanotechnology for bone materials. Wiley Interdiscip. Rev. Nanomed. Nanobiotechnol. 2009, 1, 336-351. [CrossRef] [PubMed] 
49. Sabokbar, A.; Millett, P.J.; Myer, B.; Rushton, N. A rapid, quantitative assay for measuring alkaline phosphatase activity in osteoblastic cells in vitro. Bone Miner. 1994, 27, 57-67. [CrossRef]

50. Chang, Y.L.; Stanford, C.M.; Keller, J.C. Calcium and phosphate supplementation promotes bone cell mineralization: Implications for hydroxyapatite (HA)-enhanced bone formation. J. Biomed. Mater. Res. 2000, 52, 270-278. [CrossRef] 